\title{
Atualizações sobre retinopatia diabética: uma revisão narrativa
}

\author{
Updates on diabetic retinopathy: a narrative review \\ Actualizaciones sobre la retinopatía diabética: una revisión narrativa \\ Júlia Amoroso Pereira ${ }^{1 *}$, Maurício Adriano Trentini Bertolin ${ }^{1}$, Gustavo Daun Cação Pereira ${ }^{1}$, Lucas \\ Costa Corgozinho ${ }^{1}$, Lucas Augusto da Matta Faria ${ }^{2}$, Mário Sérgio Marques Pereira².
}

\section{RESUMO}

Objetivo: Revisar na literatura a epidemiologia, os fatores de risco, a fisiopatologia, a classificação, o diagnóstico e o tratamento da retinopatia diabética. Revisão bibliográfica: $O$ diabetes mellitus é uma condição endócrino metabólica que cursa com várias complicações, dentre elas, a retinopatia diabética. Essa complicação está relacionada à alterações vasculares e está presente em grande parte dos diabéticos, podendo levar à cegueira. Para o seu desenvolvimento depende, dentre outros fatores, do tempo de evolução e dos níveis glicêmicos. Pode ser classificada em proliferativa e não proliferativa e seu diagnóstico está associado aos achados na fundoscopia. O tratamento envolve o controle glicêmico e medidas para diminuição da angiogênese. Considerações finais: Com o aumento progressivo do número de diabéticos e, consequentemente, do número de portadores de retinopatia diabética, seu estudo e abordagem são indispensáveis. A melhor compreensão do quadro, diagnóstico em tempo adequado e manejo terapêutico correto contribuem para que a retinopatia não curse com perda visual permanente.

Palavras-chave: Retinopatia diabética, Oftalmopatia, Diabetes mellitus.

\begin{abstract}
Objective: To review the literature on epidemiology, the risk factors, the pathophysiology, the classification, the diagnosis and the treatment of diabetic retinopathy. Literature review: Diabetes mellitus is a metabolic endocrine condition that leads to several complications, including diabetic retinopathy. That complication is related to vascular changes and it is present in most diabetics and can lead to blindness. For its development depends, among other factors, on the time of evolution and glycemic levels. It can be classified as proliferative and non-proliferative and its diagnosis is associated with findings on fundus of the eye exams. Treatment involves glycemic control and measures to decrease angiogenesis. Final considerations: With the progressive increase in the number of diabetics and, consequently, in the number of diabetic retinopathy patients, its study and its approach are indispensable. The best context understanding, diagnosis in a timely manner and correct therapeutic management contribute to the retinopathy do not progress with permanent visual loss.
\end{abstract}

Keywords: Diabetic retinopathy, Ophthalmopathy, Diabetes mellitus.

\section{RESUMEN}

Objetivo: Revisar la epidemiología, factores de riesgo, fisiopatología, clasificación, diagnóstico y tratamiento de la retinopatía diabética en la literatura. Revisión bibliográfica: La diabetes mellitus es una afección metabólica endocrina que conduce a varias complicaciones, incluida la retinopatía diabética. Esta

${ }^{1}$ Universidade de Franca (UNFRAN), Franca - SP. *E-mail: juliaamoroso@outlook.com

2 Santa Casa de Ribeirão Preto, Ribeirão Preto - SP. 
complicación está relacionada con cambios vasculares y está presente en la mayoría de los diabéticos y puede conducir a la ceguera. Para su desarrollo depende, entre otros factores, del tiempo de evolución y los niveles glucémicos. Se puede clasificar como proliferativa y no proliferativa y su diagnóstico se asocia con hallazgos en fondos. El tratamiento implica control glucémico y medidas para disminuir la angiogénesis. Consideraciones finales: Con el aumento progresivo en el número de diabéticos y, en consecuencia, en el número de pacientes con retinopatía diabética, su estudio y enfoque son indispensables. una mejor comprensión de la afección, un diagnóstico oportuno y un manejo terapéutico correcto contribuyen a que la retinopatía no se desarrolle con pérdida visual permanente.

Palabras clave: Retinopatía diabética, Oftalmopatía, Diabetes mellitus.

\section{INTRODUÇÃO}

O Diabetes Mellitus (DM) é uma síndrome endócrino metabólica complexa que envolve deficiência relativa ou absoluta de insulina, cursando com alterações no metabolismo de carboidratos, lipídios e proteínas. Nas últimas seis décadas o Brasil experienciou alguns movimentos de transições que ocasionaram alterações importantes no perfil das patologias que acometem grande parte da população.

Dentre as transições, observa-se o crescimento e envelhecimento populacional e destaca-se a transição nutricional, decorrente das modificações do padrão alimentar e da presença de sedentarismo, ocasionando aumento do número de pessoas com sobrepeso e obesidade e fazendo do DM uma das doenças crônicas não transmissíveis mais prevalentes nos últimos anos. Está entre as quatro principais doenças não transmissíveis, junto ao câncer, as doenças respiratóricas crônicas e as doenças cardiovasculares, segundo a Organização Mundial da Saúde (OMS) (MENDANHA DBA, et al., 2016; PÉREZ AH, et al., 2011; MENEZES LM e MORAIS NNA, 2020; SOCIEDADE BRASILEIRA DE DIABETES, 2017; RODRÍGUEZ BR, 2015).

Essa doença traz como consequências complicações macrovasculares, microvasculares e neuropáticas. Possui caráter crônico, demandando altos custos com internações e com procedimentos de média e de alta complexidade na atuação contra as complicações de longo prazo. Dentre as complicações, destaca-se a retinopatia diabética (RD), uma das principais complicações microvasculares do diabetes, que está presente, principalmente, em pacientes com curso longo da doença e com difícil controle glicêmico (PÉREZ AH, et al., 2011; ALVES AP, et al., 2014; JOST BS, et al., 2010).

Com uma evolução arrastada e progressiva, a RD pode levar à cegueira em uma porcentagem alta dos casos, sendo a principal causa de cegueira adquirida que afeta pessoas em idade produtiva. Esse fato é bastante relevante, visto que os comprometimentos visuais configuram um fator de morbidade de alto impacto social e econômico (MENDANHA DBA, et al., 2016; JOST BS, et al., 2010).

Grande parte dos diabéticos não faz exames oftalmológicos preventivos com a periodicidade recomendada, desconhecendo assim, as implicações da doença e a magnitude da sua prevenção. $O$ exame de fundo de olho constitui um método com grande praticidade e facilidade para avaliar os danos à retina, além de proporcionar dados acerca da atividade e do período de evolução da doença (VERGARA MS, et al., 2014; MENEZES LM e MORAIS NNA, 2020).

Para a prevenção da cegueira é essencial a realização de diagnóstico precoce e tratamento oportuno. Assim sendo, o papel do médico atuante na atenção primária à saúde, assim como o do médico oftalmologista é fundamental para a minimização das complicações em curso prolongado (ALVES AP, et al., 2014; RODRÍGUEZ BR, RODRÍGUEZ VR, 2015).

A RD representa uma patologia de grande relevância na área médica devido à sua crescente ascensão. Tendo em vista a grande prevalência do número de diabéticos, e, consequentemente, do número de portadores de RD e considerando suas consequências e implicações na saúde física e mental dos pacientes, assim como seu impacto socioeconômico para o sistema de saúde, se faz importante o destaque para esse tema. O presente estudo tem como objetivo revisar os dados dos últimos 10 anos acerca da epidemiologia, dos fatores de risco, da fisiopatologia, da classificação, do diagnóstico e do tratamento da RD. 


\section{REVISÃO BIBLIOGRÁFICA}

\section{Epidemiologia}

De acordo com a OMS, o número estimado de adultos convivendo com DM atualmente é de 422 milhões, e dados apontam que esse valor poderá alcançar mais de 590 milhões em 15 anos, fazendo dele uma das doenças mais prevalentes no mundo (VERGARA MS, et al., 2014; SILVEIRA VD, et al., 2018; FLOR LS e CAMPOS MR, 2017).

Dentre as nações com a maior prevalência de diabetes, o Brasil ocupa o $4^{\circ}$ lugar, totalizando cerca de 13,7 milhões de brasileiros, sendo que muitos desses pacientes não foram, ainda, diagnosticados. Foi realizado um estudo pelo Ministério da Saúde e pelo Instituto Brasileiro de Geografia e Estatística (IBGE) em 2013, em que $6,2 \%$ da população brasileira com idade superior a 18 anos referiu diagnóstico médico de DM, contabilizando $7,0 \%$ nas mulheres e $5,4 \%$ nos homens (HIRAKAWA TH, et al., 2019; SOCIEDADE BRASILEIRA DE DIABETES, 2017).

Dados apontam que a RD afeta em torno de $35 \%$ a $40 \%$ dos diabéticos, estando presente, após 20 anos de doença, em cerca de $90 \%$ dos pacientes com DM insulino dependentes e em aproximadamente $60 \%$ dos pacientes com DM não insulino dependentes (SILVEIRA VD, et al., 2018; ALVES AP, et al., 2014; MENDANHA DBA, et al., 2016).

No decorrer do tempo aproximadamente metade dos diabéticos desenvolvem RD, com efeitos que vão desde a diminuição parcial da acuidade visual até a cegueira. Estima-se que o paciente diabético possua uma probabilidade 29 vezes maior de apresentar perda visual do que um paciente não diabético.

Todas essas alterações ocasionam cerca de $7,5 \%$ das causas de incapacidade para o trabalho em adultos e, segundo a OMS, a RD é a causa de cerca de $5 \%$ dos 37 milhões de deficientes visuais presentes no mundo (JOST BS, et al., 2010; MENEZES LM e MORAIS NNA, 2020; RODRÍGUEZ BR, 2015).

\section{Fatores de risco}

Existem vários fatores de risco e de proteção relacionados às complicações retinianas em diabéticos. 0 fator mais importante relacionado à gravidade e prevalência da RD é o tempo de evolução do DM, seguido por níveis glicêmicos elevados. Existe associação entre o controle inadequado da pressão arterial e 0 surgimento de RD, assim como, a nefropatia diabética pode causar anormalidades que favorecem 0 desenvolvimento da retinopatia (PÉREZ AH, et al., 2011).

O tabagismo leva à hipóxia retiniana, aumentando a retinopatia. Segundo alguns autores, o transplante renal e de pâncreas em diabéticos aceleram o desenvolvimento da RD. Existe relação entre a elevação do perfil lipídico com o desenvolvimento de exsudatos duros e a progressão da retinopatia (PÉREZ AH, et al., 2011).

Estão associadas, também, ao desenvolvimento da retinopatia, a dieta do paciente, a existência de aterosclerose de artérias carótidas e oftálmicas internas, anemia ferropriva, grau de escolaridade, tipo de ocupação, prática de atividade física, presença de bulimia em mulheres jovens portadoras de DM tipo 1 e infecção pelo vírus da imunodeficiência humana (HIV) (PÉREZ AH, et al., 2011).

\section{Fisiopatologia}

A presença de hiperglicemia prolongada, comum ao DM, gera uma série de eventos que afetam o fluxo sanguíneo nos vasos da retina, visto que pequenos vasos sanguíneos são expostos ao dano causado pelo excesso de glicose no organismo.

Os mecanismos geradores de dano celular estão relacionados ao acúmulo de sorbitol intracelular e de produtos da glicação avançada, apuramento excessivo de isoformas da proteína quinase $C$ (PKC) e presença de estresse oxidativo. $O$ fator desencadeante da RD está relacionado a hipóxia tecidual associada à perda de autorregulação dos vasos retinianos (SILVEIRA VD, et al., 2018; HIRAKAWA TH, et al., 2019; MENDANHA DBA, et al., 2016). 
Essas alterações levam à formação de microaneurismas, perda de células endoteliais e de pericitos, células com função de reparação e suporte ao endotélio capilar; o que gera um dano a barreira hematorretiniana, podendo provocar isquemia e aumento da permeabilidade vascular. Todos esses eventos podem gerar neovascularização, edema macular, além do aumento na produção de fator de crescimento endotelial vascular (VEGF) e de outras citocinas inflamatórias. O VEGF recruta células de defesa para vasos retinianos, produzindo ainda mais citocinas (SILVEIRA VD, et al., 2018).

O estresse oxidativo, a interleucina 6 (IL-6) e o VEGF, desempenham uma função importante na patogênese da RD, principalmente na forma proliferativa da doença. Dentre outros fatores de crescimento e citocinas inflamatórias, destaca-se o fator de crescimento de fibroblasto básico (FCFb), o fator de necrose tumoral alfa (TNF- $\alpha$ ), a interleucina 1 beta (IL-1 $\beta$ ), o fator de crescimento do hepatócito (HGF), a proteína quimioatraente de monócitos-1 e a interleucina 8 (IL-8).

Dentre esses, os que parecem desempenhar um papel mais enérgico nas alterações conformacionais nas junções estreitas das células endoteliais da retina, aumentando, assim, a permeabilidade vascular e consequente quebra da barreira hematorretiniana, são a IL-6 e o VEGF (BOZKURT E, et al., 2019).

A primeira alteração na RD é o aparecimento de microaneurismas, visualizados como pequenos pontos hemorrágicos no fundo de olho. Esses microaneurismas associam-se, muitas vezes, a hemorragias retinianas, microssegmentos e veias com dilatações e tortuosidades e exsudatos duros; proveninetes do extravasamento crônico de vasos retinianos e de áreas isquêmicas (SILVEIRA VD, et al., 2018).

Os achados na realização de fundoscopia seguem um padrão progressivo, que vai da retinopatia leve, designada pelo aumento da permeabilidade vascular, até a forma moderada a grave, representada pela oclusão vascular e com posterior proliferação e cicatrização (MENDANHA DBA, et al., 2016).

Exercendo um papel significativo na fisiopatologia da RD, a Hipertensão Arterial Sistêmica (HAS) atinge duas vezes mais a população diabética. $O$ aumento da pressão arterial aumenta o extravasamento da rede vascular, incrementando a filtração de proteínas plasmáticas pelo endotélio e reposição na membrana basal capilar, sustentando assim, danos vasculares e isquemia retiniana ainda maiores (MENDANHA DBA, et al., 2016).

Alguns estudos mostram que o DM eleva a apoptose de células neurais, resultando em perda cumulativa com subsequente neurodegeneração. Estudos envolvendo tomografia de coerência óptica (OCT) e eletrofisiologia demonstaram disfunções anatômicas e alterações consideráveis nas camadas superiores da retina, previamente a observações de alterações vasculares (MALLMANN F e CANANI LH, 2019).

\section{Classificação}

A RD pode ser classificada em proliferativa e não proliferativa. O que caracteriza a forma proliferativa é a existência de vasos sanguíneos alterados a partir da retina e do nervo óptico. Na presença de neovascularização há um risco aumentado de ocorrer hemorragia vítrea e retiniana, além de descolamento de retina. Este último pode ocorrer se houver tração da rede fibrovascular proliferada (SILVEIRA VD, et al., 2018; JIMENÉZ-BÁEZ MV, et al., 2015).

A retinopatia diabética não proliferativa (RDNP) pode ir desde uma graduação leve até grave, podendo progredir, então, para a forma proliferativa, quando se estabelece neovascularização advinda dos vasos retinianos ou do disco óptico (SILVEIRA VD, et al., 2018; JIMENÉZ-BÁEZ MV, et al., 2015).

A retinopatia diabética proliferativa (RDP) pode ser classificada em inicial, de alto risco e grave. Essa forma de retinopatia pode ou não ser precedida pela forma não proliferativa. A chance de progressão de formas leves para formas mais graves, em 5 anos, é de cerca de $75 \%$. Além disso, há risco de perda visual aguda reversível, que pode ocorrer quando há hemorragia intravítrea, ocorrendo bloqueio no alcance dos raios luminosos até a retina, porém, costumam ser reabsorvidos, reestabelecendo-se a visão de maneira espontânea; e perda visual definitiva, quando há descolamento de retina ou isquemia macular. Se o tratamento não for realizado, aproximadamente metade dos pacientes com RDP de alto risco apresentarão perda visual severa em cerca de 5 anos (SILVEIRA VD, et al., 2018; GRAUSLUND J, 2017). 


\section{Diagnóstico}

As alterações oftálmicas causadas pelo diabetes se manifestam com poucos sintomas até levarem à perda visual e o risco de cegueira pode ser reduzido a porcentagens inferiores a $5 \%$ quando o diagnóstico é realizado em tempo adequado e o manejo é efetuado corretamente, antes que alterações irreversíveis possam se instalar (SILVEIRA VD, et al., 2018; HIRAKAWA TH, et al., 2019).

Recomenda-se que o primeiro exame do fundo de olho no diabético do tipo 1 seja realizado após a puberdade ou cinco anos após o diagnóstico, visto que a RD dificilmente é observada antes desse período. Já nos diabéticos do tipo 2, recomenda-se a realização logo após o diagnóstico pois normalmente não se pode definir precisamente quando o DM foi instalado e a retinopatia já pode estar presente no momento do diagnóstico (SILVEIRA VD, et al., 2018; PRETI RC, et al., 2010).

A frequência das avaliações pode ser anual, semestrais e até mesmo mensais, de acordo com a gravidade da retinopatia e tendo em vista que os diabéticos apresentam um risco maior de desenvolver outras patologias oftalmológicas, como catarata e glaucoma. Para as gestantes portadoras de algum tipo de diabetes, indicase avaliações trimestrais devido ao maior risco de progressão da doença (SILVEIRA VD, et al., 2018; JOST BS, et al., 2010).

Os critérios diagnósticos e caracterização das oftalmopatias diabéticas são fundamentados nas definições e achados. Os achados fundoscópicos iniciais da RD são compostos por microaneurismas que podem ser visualizados tanto na mácula, quanto na periferia. O padrão ouro para o diagnóstico da RD é a fundoscopia sob midríase.

A realização de angiografia fluoresceínica pode ajudar na identificação de áreas com extravasamento de vasos sanguíneos e locais com isquemia capilar (SILVEIRA VD, et al., 2018). Apesar de todas as informações, a aderência a essas recomendações é baixa, com estudos mostrando que apenas $30 \%$ dos diabéticos seguem o regime de acompanhamento oftalmológico (JOST BS, et al., 2010).

\section{Tratamento}

O tratamento clínico da RD tem por objetivo estabilizar o sistema vascular e reduzir de forma considerável o risco de perda visual. Esse tratamento gira em torno da terapia primária, que inclui a intervenção multidisciplinar para o controle sistêmico, de forma rigorosa, dos níveis glicêmicos, lipídicos, e pressórico; e a terapia secundária, que inclui a administração de medicamentos anti-angiogênicos via injeção intraocular na cavidade vítrea, associados ou não à fotocoagulação a laser da retina, e tratamento cirúrgico.

Estudos demonstram que a cada $1 \%$ de redução da hemoglobina glicada ocorre uma redução no risco de desenvolvimento e progressão da retinopatia de $35 \%$ e de $39 \%$, respectivamente (JOST BS, et al., 2010; MALLMANN F e CANANI LH, 2019; HIRAKAWA TH, et al., 2019; JÚNIOR OOM, 2015; SABROSA NA, et al., 2013).

A fotocoagulação a laser, conhecida por ser um dos primeiros tratamentos para RD, tem como intuito a cauterização de microaneurismas e ablação de áreas sem perfusão capilar. A sua realização melhora a oxigenação da retina, o que ocasiona, posteriormente, níveis mais baixos de VEGF. Trabalhos demonstraram melhora em até $50 \%$ do risco de perda visual.

Apesar dos efeitos benéficos, a fotocoagulação pode apresentar alguns efeitos adversos como diminuição do campo visual periférico e cegueira noturna (SILVEIRA VD, et al., 2018; GRAUSLUND J, 2017). O uso dos medicamentos anti-VEGF, que revolucionou o tratamento de várias retinopatias, fundamenta-se no fato de que o VEGF é a molécula mais atuante na fisiopatologia da RD.

Consistem na aplicação intravítreo de anticorpos monoclonais, como ranibizumab, bevacizumab e aflibercept, tendo como finalidade a supressão da ação do fator de crescimento neovascular, atenuando a angiogênese e a permeabilidade capilar. Vem sendo utilizada como terapia adjuvante previamente a aplicação do laser e diversos estudos comprovam a sua eficácia, tanto quando usada de maneira isolada, quanto usada em associação a outros tratamentos, como a fotocoagulação a laser. 
Estudos atuais comprovam a efetividade e segurança a curto prazo do uso desses medicamentos no tratamento da RDP e estudos abrangendo resultados a longo prazo dessa terapia serão importantes na decisão da melhor estratégia terapêutica (SILVEIRA VD, et al., 2018; GRAUSLUND J, 2017).

Outra opção terapêutica são as injeções intravítreo de corticóides, como a dexametasona. Essa opção apresenta efetividade na diminuição do edema, agindo na atividade e expressão de algumas citocinas inflamatórias, além de ajudar a manter a barreira hematorretiniana. $O$ inconveniente de seu uso é representado pela elevada incidência de efeitos adversos, como desenvolvimento de catarata, aumento da pressão intraocular e endoftalmite. Contudo, o uso desses medicamentos é uma opção nos casos refratários ao uso de anti-VEGF (SILVEIRA VD, et al., 2018).

Estudos mostram que o uso dos inibidores da enzima conversora de angiotensina (IECA) podem ser benéficos para impedir a progressão da retinopatia, tendo em vista seu papel em minimizar a incidência de doença renal diabética. Seguindo os guidelines internacionais, o tratamento da RDNP deve ser iniciado nos casos leve ou moderado associado a edema macular, estando indicada a utilização de anti-VEGF ou de fotocoagulação focal a laser.

Nos casos sem edema macular, o tratamento com anti-VEGF ainda é discutível. O tratamento da RDP é instaurado nas formas precoces mediante fotocoagulação, e nas formas de risco elevado e graves, utiliza-se fotocoagulação com possibilidade de terapia adjuvante com anti-VEGF (PÉREZ AH, et al., 2011; SILVEIRA VD, et al., 2018).

Uma droga que apresenta larga experiência mundial quanto à eficácia, utilizada de forma "off label", é a triancinolona. Um dos fatores notáveis para sua escolha é o acesso ao tratamento, apresentando um custo mais baixo. Infere-se que seu mecanismo de ação na RD gere um efeito anti-inflamatório através da estabilização da barreira hematorretiniana, e, principalmente, redução dos mediadores inflamatórios. Alguns estudos comprovam a eficácia da triancinolona intravítrea como adjuvante à laserterapia em comparação a laserterapia isolada em portadores de RDP e edema macular (JÚNIOR OOM, 2015).

Em alguns casos, quando há descolamento de retina; hemorragia intensa no vítreo, chegando a impedir a aplicação de laser; ou mesmo quando as terapias, primária e secundária, não apresentam resposta, utilizase a vitrectomia como opção terapêutica. A sua realização promove remoção de opacidades do meio, como a hemorragia vítrea, e possibilita a liberação de trações vitreorretinianas. Ademais, a realização da fotocoagulação retiniana intraoperatória pode facilitar a estabilização do processo vasoproliferativo intraocular. A acuidade visual final após o procedimento varia bastante, contudo, a maior parte dos pacientes se beneficiam com a sua realização (SILVEIRA VD, et al., 2018; SABROSA NA, et al., 2013).

Estima-se que cerca de $5 \%$ dos pacientes com RDP, necessitam de vitrectomia, independentemente de controles glicêmico e pressórico adequados e de fotocoagulação devidamente realizada. As complicações associadas à realização de vitrectomia implicam hemorragia vítrea recorrente, glaucoma neovascular, descolamento de retina regmatogênico e rubeose, que corresponde a neovascularização da íris. Pode-se utilizar injeções de anti-VEGF no pré-operatório para minimizar as complicações (SILVEIRA VD, et al., 2018; SABROSA NA, et al., 2013).

\section{CONSIDERAÇÕES FINAIS}

A RD configura uma causa relevante de perda visual, apresentando uma prevalência crescente. Os pacientes que possuem a doença por muito tempo ou aqueles que demandam maior uso de medicações, apresentam risco aumentado de lesão retiniana grave e perda funcional. Esse contexto é alarmante, visto que cerca de $50 \%$ dos diabéticos irão desenvolver algum estágio de RD no decorrer de suas vidas. A realização de diagnóstico precoce e intervenções apropriadas, tanto com terapias farmacológicas, quanto cirúrgicas, quando indicadas, é fundamental para que a perda visual seja evitada na maior parte dos casos. Além disso, é de grande importância a conscientização dos pacientes a respeito da doença e da relevância do controle glicêmico e de outras medidas em seu desfecho. O manejo adequado e individualizado visa potencializar a preservação visual e reduzir os efeitos colaterais. 


\section{REFERÊNCIAS}

1. ALVES AP, et al. Retinopatia em pacientes hipertensos e/ou diabéticos em uma unidade de saúde da família. Revista Brasileira de Oftalmologia, 2014; 73(2): 108-111.

2. BOZKURT E, et al. Correlation of the aqueous humor total antioxidant capacity, total oxidant status, and levels of IL6 and VEGF with diabetic retinopathy status. Arquivos Brasileiros de Oftalmologia, 2019; 82(2): 136-140.

3. FLOR LS, CAMPOS MR. Prevalência de diabetes mellitus e fatores associados na população adulta brasileira: evidências de um inquérito de base populacional. Revista Brasileira de Epidemiologia, 2017; 20(1): 16-29.

4. GRAUSLUND J. Vascular endothelial growth factor inhibition for proliferative diabetic retinopathy: Et tu, Brute? Acta Ophthalmologica, 2017; 95: 757-758.

5. HILGERT GR, et al. Uso de retinografo portatil como ferramenta no rastreamento de retinopatia diabetica. Revista Brasileira de Oftalmologia, 2019; 78 (5): 321-326.

6. HIRAKAWA TH, et al. Conhecimento dos pacientes diabéticos usuários do Sistema Único de Saúde acerca da retinopatia diabética. Revista Brasileira de Oftalmologia, 2019; 78 (2): 107-111.

7. JIMENÉZ-BÁEZ MV, et al. Early diagnosis of diabetic retinopathy in primary care. Colombia Médica, 2015; 46(1): 1418.

8. JOST BS, et al. Prevalência de retinopatia diabética na população portadora de diabetes mellitus tipo 2 do município de Luzerna - SC. Arquivos Brasileiros de Oftalmologia, 2010;73(3): 259-265.

9. JÚNIOR OOM. Triancinolona no tratamento da retinopatia diabética. Revista Brasileira de Oftalmologia, $2015 ; 74$ (4): 201-202.

10. MALLMANN F, CANANI LH. Intravitreal neurodegenerative and inflammatory mediators in proliferative diabetic retinopathy. Arquivos Brasileiros de Oftalmologia, 2019; 82(4): 275-282.

11. MENDANHA DBA, et al. Fatores de risco e incidência da retinopatia diabética. Revista Brasileira de Oftalmologia, 2016; 75 (6): 443-446.

12. MENEZES LM, MORAIS NNA. Achados de fundoscopia de pacientes diabeticos e/ou hipertensos. Revista Brasileira de Oftalmologia, 2020; 79(1): 28-32.

13. PÉREZ AH, et al. Factores de riesgo en el desarrollo de la retinopatía diabética. Revista Cubana de Oftalmología, $2011 ; 24(1): 86-99$.

14. PRETI RC, et al. Relationship between diabetic retinopathy severity and the timespan between the endocrinopathy diagnosis and the first ophthalmic examination. Arquivos Brasileiros de Oftalmologia, 2010; 73(3): 240-243.

15. RODRÍGUEZ BR. Prevención de ceguera por retinopatía diabética: ¿dónde estamos? Revista Cubana de Oftalmología, 2015; 28(1): 119-128.

16. RODRÍGUEZ BR, RODRÍGUEZ VR. Retinopatía diabética, una epidemia prevenible y tratable. Revista Cubana de Oftalmología, 2015; 28(1): 1-2.

17. SABROSA NA, et al. Tratamento cirúrgico da retinopatia diabética. Revista Brasileira de Oftalmologia, $2013 ; 72$ (3): 204-209.

18. SILVEIRA VD, et al. Atualizações no manejo de retinopatia diabética: revisão de literatura. Acta Medica - Ligas Acadêmicas, 2018; 39(1): 293-306.

19. SOCIEDADE BRASILEIRA DE DIABETES. Diretrizes da Sociedade Brasileira de Diabetes 2017-2018. Editora Clannad, 2017; 1: 12-13.

20. VERGARA MS, et al. Achados epidemiológicos e alterações oftalmológicas em diabéticos atendidos em hospital geral secundário. Revista Brasileira de Oftalmologia, 2014; 73 (3): 167-170. 